\title{
La comunidad en los tiempos de la Comunidad: bienestar en las Comunidades Nativas asháninkas
}

La communauté au temps de la Comunidad: bien-être dans les Comunidades Nativas Asháninka

Community in the times of la Comunidad: Wellbeing in Ashaninka Native

Communities

\section{Juan Pablo Sarmiento Barletti}

\section{OpenEdition \\ Journals}

Edición electrónica

URL: https://journals.openedition.org/bifea/7904

DOI: 10.4000/bifea.7904

ISSN: 2076-5827

Editor

Institut Français d'Études Andines

Edición impresa

Fecha de publicación: 1 abril 2016

Paginación: 157-172

ISSN: 0303-7495

Referencia electrónica

Juan Pablo Sarmiento Barletti, «La comunidad en los tiempos de la Comunidad: bienestar en las Comunidades Nativas asháninkas», Bulletin de l'Institut français d'études andines [En línea], 45 (1)| 2016, Publicado el 08 abril 2016, consultado el 21 septiembre 2021. URL: http:// journals.openedition.org/bifea/7904 ; DOl: https://doi.org/10.4000/bifea.7904 


\title{
La comunidad en los tiempos de la Comunidad: bienestar en las Comunidades Nativas asháninkas
}

\author{
Juan Pablo Sarmiento Barletti*
}

\begin{abstract}
Resumen
El presente artículo estudia las diferencias críticas que los asháninkas, miembros de la sociedad indígena más grande de la Amazonía peruana, encuentran entre la Comunidad Nativa como una entidad política, y la comunidad compuesta de asháninka sanori («personas legítimas») que ellos construyen en la vida cotidiana. Aunque estos dos tipos de «comunidad» parezcan contradictorias, la estructura y reglas asociadas con la Comunidad Nativa funcionan a favor del kametsa asaiki («vivir bien juntos»), el ethos de bienestar asháninka. De hecho, la Comunidad Nativa presenta un marco de normas, reglamentos, y derechos ante el Estado peruano que los asháninkas utilizan de manera estratégica para defender al kametsa asaiki. Esta defensa es especialmente importante en el contexto del proceso asháninka de reconstrucción después del conflicto interno peruano (1980-2000), la imposición de proyectos extractivos en su territorio, y las transformaciones en sus relaciones sociales, al pasar de vivir en grupos de parentesco pequeños a aldeas de cientos de personas.
\end{abstract}

Palabras clave: Comunidad Nativa, bienestar, asháninka, Amazonía

\section{La communauté au temps de la Comunidad: bien-être dans les Comunidades Nativas Asháninka}

\section{Résumé}

Cet article aborde les sérieuses différences que les Asháninka — la société indigène d'Amazonie péruvienne qui compte le plus grand nombre de membres — rencontrent entre la Comunidad Nativa

Profesor en Antropología, Durham University. E-mail: juan-pablo.sarmiento-barletti@durham.ac.uk Agradezco los comentarios del evaluador anónimo del BIFEA, así como los comentarios y ayuda editorial de José Barletti Pasquale. Todos los errores ortográficos y de interpretación restantes son míos. 
comme entité politique et asháninka sanori (« les vraies personnes »), la communauté qu'ils construisent au quotidien. Bien que ces deux types de «comunidad» apparaissent contradictoires, la structure et les règles associées à la Comunidad Nativa fonctionnent autour de la notion de kametsa asaiki (« vivre bien ensemble »), l'ethos de bien-être asháninka. De fait, la Comunidad Nativa est un marqueur de normes, de règlements et de droits face à l'État péruvien que les Asháninka utilisent de manière stratégique pour défendre leur kametsa asaiki. Défendre leur kametsa asaiki est particulièrement important dans le contexte du processus asháninka de reconstruction après le conflit interne péruvien (1980-2000), l'imposition de projets extractivistes sur leur territoire et les transformations de leurs relations sociales en passant d'une organisation en petits groupes de parenté à des communautés de plusieurs centaines de personnes.

Mots-clés : Comunidad Nativa, bien-être, Ashaninka, Amazonie

\title{
Community in the times of la Comunidad: Wellbeing in Ashaninka Native Communities
}

\begin{abstract}
This article addresses the critical differences that Ashaninka people, members of the largest indigenous society of the Peruvian Amazon, find between the Comunidad Nativa as a political entity and the community of Ashaninka sanori ("real people") in which they live in on a daily basis. Although seeming to clash, the structure and rules associated with the Comunidad Nativa work in favour of their ethos for well-being (kametsa asaiki). In fact, the Comunidad Nativa presents a framework of rules, regulations, and rights that are used strategically by Ashaninka people to defend kametsa asaiki. This defense is especially important in the context of the Ashaninka reconstruction process in the wake of the Peruvian internal war (1980-2000), the imposition of extraction projects in their territory, and the transformation of their social relations following the shift from living in small dispersed kin-based groups to one of living in large villages.
\end{abstract}

Keywords: Comunidad Nativa, well-being, Ashaninka, Amazonia

\section{INTRODUCCIÓN: LA COMUNIDAD Y LA COMUNIDAD}

En este artículo reviso las diferencias críticas que los asháninkas, miembros de la sociedad indígena más grande de la Amazonía peruana, encuentran entre Comunidad Nativa como una entidad política con un título de propiedad colectiva y la comunidad compuesta por individuos asháninka sanori («persona legítima»), que ellos crean en sus interacciones cotidianas y que ocupa el territorio titulado. Esta revisión está basada en siete años de trabajo de campo etnográfico con grupos asháninkas en los valles de los ríos Bajo Urubamba, Ene, Tambo, y Ucayali. Con ella, me uno a las lecturas críticas que se han venido haciendo sobre la Comunidad Nativa desde la década de los años 1980. Estas críticas, que discutiré más abajo, demuestran cómo la Ley de Comunidades Nativas¹ ha llevado a la imposición de

1 Decreto-Ley n. ${ }^{\circ}$ 22175-Ley de Comunidades Nativas y de Desarrollo Agrario de la Selva y de Ceja de Selva. Esta ley fue introducida en 1974 durante el gobierno del General Juan Velasco Alvarado. 
conceptos y estructuras políticas que no corresponden a la organización social de las sociedades indígenas amazónicas.

Sin embargo, al considerar estas críticas desde la realidad vivida por mis colaboradores ${ }^{2}$ asháninkas, busco demostrar la creatividad con la que ellos han aprovechado las estructuras que les impone el Estado peruano por medio de la Ley de Comunidades Nativas, para retomar y reforzar su proyecto de bienestar (kametsa asaiki, «vivir bien juntos») dentro de las complicaciones del contexto de posguerra interna ${ }^{3}$ creciente extractivismo en el que viven ${ }^{4}$. Al proponer esto, sostengo que la Comunidad Nativa se ha convertido en una parte clave del actual proyecto asháninka que concierne la búsqueda del kametsa asaiki.

Voy a considerar primero la crítica que se ha hecho a la Comunidad Nativa desde la década de 1980, para luego pasar a revisar el caso específico de los asháninkas, y las oportunidades que la Comunidad Nativa les da para poder formar una «Comunidad de similares» (Overing, 2003) en la vida cotidiana. La forma tan positiva en la que mis colaboradores asháninkas hablan de la Comunidad Nativa nos presenta una especie de enigma etnográfico. En efecto, como discutiré más abajo, los estudiosos de los diferentes grupos asháninkas y ashéninkas escriben que su forma de asentamiento tradicional se caracteriza por patrones dispersos, en pequeños grupos basados en parentesco (ver, por ejemplo, Hvalkof \& Veber, 2005; Killick, 2005; Pimenta, 2006). La preferencia por este tipo de organización ha llevado a los estudiosos de esta sociedad indígena a describir la vida social asháninka como una en la que la fragmentación y ruptura son la norma, y la unidad social y la acción colectiva son solo transitorias. En gran parte, este tipo de organización se explica por la falta de mecanismos tradicionales para resolver conflictos dentro de la sociedad asháninka (ver Santos-Granero, 2000). Esta dificultad para resolver conflictos se complica aún más en el contexto de posguerra, ya que las aldeas asháninkas llegan a tener cientos de habitantes que

2 Como en el resto de mis publicaciones, en este artículo hago referencia a mis «colaboradores» o «interlocutores» asháninkas. Al evitar el término «informante» para referirme a ellos, busco resaltar su rol activo en mis investigaciones, en las que son mucho más que simples objetos de estudio antropológico.

3 En este artículo no trato en detalle la experiencia asháninka del conflicto interno en el Perú (19802000). Para saber más del tema, ver Sarmiento Barletti (2011) y Caruso (2012). Las muertes asháninkas durante el conflicto se estiman entre 6000 y 8000 (de casi 70000 muertes en todo el Perú), y 51 de las 66 aldeas de los valles de Ene y Tambo fueron destruidas. Esto llevó al desplazamiento de 10000 personas asháninkas. La magnitud de la guerra en términos de muertos y desaparecidos, la destrucción de aldeas enteras y la circulación de historias sobre los excesos de los senderistas dejaron a los asháninkas viviendo en un estado de guerra hasta mucho después del final oficial del conflicto en 2000 (ver CVR, 2002).

4 Tampoco trataré en detalle el impacto de la expansión de la frontera extractivista en las aldeas asháninka de la selva central (ver Sarmiento Barletti, 2016b). El territorio asháninka recibe el impacto de empresas extractoras de gas (PlusPetrol) y petróleo (Repsol), así como de grandes madereras (Consorcio Amazónico), y planes para represas hidroeléctricas (Pakitzapango y Tambo 40). Mis colaboradores asháninka explican que, sumado a la violencia del conflicto interno, este impacto ha llevado a un rompimiento de las relaciones sociales positivas que los asháninkas tenían con aipatiste («nuestro territorio») antes de la guerra, lo cual ha llevado a una escases de animales de caza y una disminución de la productividad de sus chacras. 
no están necesariamente relacionados en términos de parentesco y muchos de ellos estuvieron en bandos opuestos durante el conflicto.

Basado en estas dificultades, este artículo quiere explicar cómo los cambios estratégicos que la Comunidad Nativa permite entre las identidades de comunero (una identidad política) y asháninka (una identidad cotidiana de parentesco), han permitido que mis colaboradores puedan resolver conflictos sin destruir la frágil convivialidad 5 que tanto les cuesta construir en su vida diaria. Estos cambios estratégicos demuestran que los asuntos oficiales de la Comunidad Nativa manejados por su jefe, al estar basados en un poder conferido por el Estado peruano, entidad exterior a la vida social asháninka, se entienden como separados de los procesos diarios de parentesco y de la búsqueda del kametsa asaiki, por más que en la práctica todos estos estén interconectados.

\section{LA COMUNIDAD NATIVA}

El Decreto Legislativo (DL) 20653, sobre la «Ley de Comunidades Nativas y Promoción Agropecuaria de las Regiones de Selva y Ceja de Selva», fue promulgado en 1974 durante el gobierno militar del General Juan Velasco. El espíritu original del DL 20653 reconocía permitía reconocer la continuidad histórica del derecho de los pueblos indígenas sobre los territorios que ocupaban en la Amazonía. Este, además, le daba a los pueblos indígenas el derecho inalienable e inembargable a la propiedad colectiva sobre el territorio de las Comunidades Nativas que les serían tituladas por el Estado. Sin embargo, 4 años después, durante el gobierno militar del General Morales Bermúdez, el DL 20653 fue derogado por el DL 21147, la «Ley de Comunidades Nativas y Desarrollo Agrario de las Regiones de Selva y Ceja de Selva». La diferencia clave entre estas dos leyes no solo está en el cambio de «Promoción Agropecuaria» a «Desarrollo Agrario», sino en la introducción de un nuevo artículo. Este nuevo artículo dice:

La parte del territorio de las Comunidades Nativas que corresponda a tierras con aptitud forestal, les será cedida en uso y su utilización se regirá por la legislación sobre la materia (DL 21147, Art. 11).

Este cambio terminó con el espíritu original del DL 20653, ya que los bosques nunca regresaron a ser propiedad de las Comunidades.

Desde los años 1980, las críticas a la Ley de Comunidades Nativas se presentan como una herramienta encubierta para el control de los pueblos indígenas amazónicos y sus territorios tradicionales. No voy a profundizar mucho en esto ya que ha sido hecho de manera más detallada por otros estudiosos del tema (ver, por ejemplo, Gray, 1997; 1998; Surralles \& García, 2005; Chirif \& García, 2007). Las críticas sobre el tema cubren diferentes aspectos de la Ley de

5 Aquí utilizo el término «convivialidad» siguiendo la aplicación analítica que hacen Joanna Overing (ver la introducción a Overing \& Passes, 2000), y sus estudiantes como Luisa Elvira Belaunde (2000) y Peter Gow (2000). 
Comunidades Nativas que van desde la definición capitalista que la ley le da a los recursos naturales6, hasta lo complicado de los trámites que se requieren para lograr la titulación de una Comunidad, los cuales pueden tener hasta veintiséis pasos. Cabe resaltar que hay estudios sobre las razones por las cuales diferentes grupos asháninkas han buscado titular sus Comunidades. Mientras que Hvalkof (1998) y Hvalkof \& Veber (2005) recalcan que estos procesos se dan para defender su territorio e identidad étnica, Killick (2008) argumenta que la motivación principal para su titulación es que el Ministerio de Educación les dote de un profesor para sus escuelas. Sin embargo, estos análisis no nos dicen mucho sobre la forma en que los diferentes grupos asháninkas entienden a la figura de la Comunidad Nativa una vez que ya han logrado titular una parte de su territorio.

La Comunidad Nativa fue una figura legal muy complicada desde sus inicios, ya que se basaba en un modelo de organización social andino impuesto sobre el modelo tradicional de organización socioespacial amazónico. Estos modelos están basados en formas diferentes de pensar y relacionarse con el territorio ${ }^{7}$, y con las entidades sociales que viven dentro de este. Con el tiempo se volvió obvio que lo que la ley buscaba era encasillar a los grupos indígenas amazónicos dentro de espacios predeterminados, para así poder tomar control de las tierras que no fueron tituladas y dárselas a terceros para la explotación comercial de sus recursos naturales ${ }^{8}$. Recordemos que hasta no mucho antes que se aprobara la Ley de Comunidades Nativas, la selva peruana era considerada como «una tierra sin hombres para hombres sin tierra» (Belaúnde, 1959: 105).

Además, por medio de la Ley de Comunidades Nativas, el Estado obliga a los miembros de las Comunidades Nativas a elegir jefes quienes, al ser sus representantes legales, reciben poder autoritario sobre los recursos naturales dentro del territorio titulado de la Comunidad, además de otros tipos de interacciones con foráneos que describiré más adelante. La autoridad que recibe estos jefes choca con la conocida voluntad «en contra del estado» (Clastres, 1987 [1974]) que se ha vuelto famosa entre estas sociedades. La Comunidad Nativa trajo además un cambio en la organización física de la vida social asháninka que pasó de una organización en parentelas divididas en el bosque, a una vida en aldeas. Como nota Hanne Veber, la Comunidad Nativa presupone

un tipo de organización de la población rural en aldeas de tipo comunal, una forma de organización que es extraña para muchos grupos amazónicos (Veber, 1998: 394, mi traducción; ver también Killick, 2008).

6 Por ejemplo, el primer artículo de la ley describe que esta busca «establecer una estructura agraria que contribuya al desarrollo integral de las regiones de Selva y Ceja de Selva, a fin de que su población alcance niveles de vida compatibles con la dignidad de la persona humana».

7 Aquí sigo la distinción entre «tierra» (como un recurso económico) y «territorio» (con dimensiones sociales) que se ha hecho en la literatura sobre la Amazonía indígena peruana (por ejemplo, Chirif \& García Hierro, 2007), y discursos indígenas sobre el tema (ver, por ejemplo, Corpi, 2002: 45 in Espinosa, 2010: 249).

8 Este es un proceso que se ha dado en otros contextos y que David Harvey (2003) describe como «acumulación por desposesión». 
Este cambio fue reforzado por el Sinamos (Sistema Nacional de Movilización Social), que tuvo «un rol importante en la promoción de asentamientos nucleados (...) reforzando el nuevo modelo» (Santos-Granero \& Barclay, 1998: 243, mi traducción).

Si bien el proceso de titulación de las Comunidades se volvió un proceso de «despojo institucionalizado» (Chirif, 1980; ver también Chirif, 1982), también se presentó como una oportunidad para que los grupos indígenas que se veían con cada vez menos territorio, debido a las invasiones de colonos andinos, pudieran asegurar sus derechos sobre «lo que queda[ba]» (Barclay \& Santos Granero, 1980: 45) antes de perderlo todo. Esto significó que gran parte del territorio tradicional de las sociedades indígenas de la Amazonía peruana haya quedado fuera de las áreas tituladas como Comunidades Nativas. Al pasar el tiempo, «lo que queda[ba]» continuó siendo cada vez menos. Con los cambios hechos a la Ley de Comunidades Nativas en la Constitución de 1993 durante el régimen de Alberto Fujimori, los territorios titulados perdieron las características de inalienable e inembargable. Además, se añadió un artículo estableciendo que el Estado podía tomar las tierras que este consideraba en estado de «abandono» para dárselas a terceros. A pesar de los convenios internacionales sobre derecho indígena que el Estado peruano firmó a lo largo de los años (por ejemplo, el Convenio 169 de la Organización Internacional del Trabajo), se continuó con políticas enfocadas en el despojo de territorio indígena para la extracción de recursos naturales. Esta política de facilitar las inversiones extractivas en el Perú llegó a un punto álgido en el gobierno de Alan García, durante el Paro Nacional Amazónico, trágicamente conocido a nivel mundial ${ }^{9}$ por la represión de los grupos indígenas por parte de las fuerzas del gobierno en las inmediaciones de la ciudad de Bagua el 5 de junio de 2009.

A pesar de las imposiciones que la Comunidad trajo consigo, mis colaboradores asháninkas destacan que ésta es clave para la consecución de lo que ellos llaman kametsa asaiki («vivir bien juntos»), su ethos de bienestar. El énfasis que mis colaboradores asháninkas le dan al kametsa asaiki en la vida diaria se debe a que este ethos lleva a la fabricación comunal del asháninka sanori («persona legitima»). Mucho se ha escrito sobre cómo la humanidad en la Amazonía indígena no es un hecho biológico, sino el resultado de un proceso sociológico que, con mucha dificultad, se entabla en la vida diaria (ver, por ejemplo, Taylor 1996). Entonces, el asháninka sanori no nace, sino se fabrica de manera relacional a través de tres conjuntos de conocimientos que están interrelacionados ${ }^{10}$. El primero de estos conjuntos es el control de las emociones antisociales (como la ira, los celos, y la avaricia), y la práctica cotidiana de las emociones que ellos consideran como socialmente constructivas (como el amor, la felicidad y la pena). El segundo

9 Algunos sectores de la prensa internacional lo llamaron «el Tiananmén amazónico» (http://www. independent.co.uk/news/world/americas/images-of-amazons-tiananmen-1708990.html)

10 El término asháninka es relacional de por sí ya que la «a» es el plural inclusivo en idioma asháninka. El etnónimo se traduce al español como «la gente» o «los paisanos». 
conjunto tiene que ver con una ética de trabajo ya que los ashaninka sanori son seres socialmente productivos que, al expresar tanto su generosidad como su preocupación por las necesidades de los otros, comparten el producto de su trabajo, están dispuestos a recibir el producto del trabajo de otros y compartir en la felicidad que estos intercambios generan. El tercer conjunto está asociado con las relaciones de cuidado practicadas por aquellos que se relacionan como ashaninka sanori, con énfasis en la comensalía y el disfrute de las sustancias que ellos consideran como productoras de sociabilidad (como la ayahuasca), al mismo tiempo que evitan las sustancias negativas. Todo esto se relaciona con el arduo trabajo que se dedica a la creación de los «cuerpos fuertes» y «caras felices» asháninkas en la vida cotidiana.

En las siguientes secciones exploro las complicaciones que la Comunidad Nativa presenta para con las nociones tradicionales asháninkas de organización espacial y de liderazgo. Como veremos, si seguimos estrictamente el corpus etnográfico sobre cómo los asháninkas organizan su vida social físicamente, y la autoridad que les confieren a sus líderes, nos puede sorprender que mis colaboradores asháninkas hayan podido constituirse exitosamente en aldeas hoy en día, y consideren esta forma de organización como la más ideal para su proyecto de bienestar. ¿Cómo, desde su punto de vista, el vivir en grupos grandes, y bajo las estructuras de Comunidad Nativa, deja de ser una imposición y se convierte en una precondición para el kametsa asaiki?

\section{LA ORGANIZACIÓN FÍSICA DE LA VIDA SOCIAL ENTRE LOS ASHÁNINKAS}

Históricamente, los patrones de asentamiento asháninka se han caracterizado por ser pequeños grupos familiares que viven a cierta distancia cada uno del otro en el bosque. Gerald Weiss (1975) considera que estos grupos familiares son las más grandes unidades políticas estables en la sociedad asháninka. Estas «pequeñas agrupaciones de casas constan de (...) entre una y seis familias nucleares ligadas por lazos de parentesco (...) que se caracterizan por un alto grado de reciprocidad y cooperación económica entre las diferentes unidades domésticas que las componen» (Pimenta, 2006: 3, mi traducción).

Los estudiosos de los diferentes grupos asháninkas han explicado esta organización de asentamiento disperso en términos que van desde las limitaciones de recursos naturales (Denevan, 1974) hasta el cumplimiento de una vida pacífica (Killick, 2005). De hecho, gran parte de esta línea analítica ha querido entender cómo los asháninkas hacen para organizarse en grupos grandes para enfrentarse con un enemigo común, cuando en el día a día su organización social parece estar tan atomizada. Søren Hvalkof \& Hanne Veber (2005) nos dicen que cuando este tipo de alianzas ocurren, la acción colectiva tiende a ser de corta duración ya que las familias regresan a vivir de manera separada una vez que la amenaza ha disminuido. Estas características han llevado a estos antropólogos a describir la 
vida social asháninka como una en la que la fragmentación y la ruptura son la norma, y la unidad social y la acción colectiva son solo transitorias.

El cambio en la organización física de la vida social asháninka que vino con la Comunidad Nativa se reforzó en la posguerra como una estrategia para poder defenderse mejor de futuros ataques de Sendero Luminoso. Les permitió también defenderse de las presiones sobre sus vidas y su territorio por parte del Estado peruano y de las industrias de hidrocarburos por medio de proyectos de extracción de gas y petróleo, así como proyectos para construir represas en sus ríos y más carreteras. Mis colaboradores asháninkas explican que mientras que sus ancestros comunes (acharineite, «nuestros abuelos», o «los antiguos») vivían separados, ellos tuvieron que unirse para poder vivir tranquilamente:

Nuestros abuelos no vivían en [aldeas], vivían en familia nomás, separados en el monte (...) así vivían antes, separados, bien lejos y a veces se visitaban para tomar masato (...) Pero ya no vivimos así porque nuestro territorio ya no es amplio como antes. Ahora tenemos a los colonos cerca (...) hemos tenido también a los [senderistas], a la empresa [de hidrocarburos], a los madereros (...) entonces hemos hecho nuestra Comunidad para vivir tranquilos y defendernos.

Con estos cambios, los asentamientos locales asháninkas pasaron de ser parentelas de entre treinta y cincuenta habitantes, a constituir aldeas de entre doscientos y trescientos habitantes en promedio, aunque muchas sobrepasan los quinientos habitantes. La organización física de estas aldeas refleja la de los centros urbanos amazónicos, con un espacio central público (una cancha de futbol en el caso de las Comunidades), junto al cual se ubican otros edificios públicos como una casa comunal (la cual normalmente tiene una bandera peruana), una escuela, y una posta médica. Las casas normalmente se construyen una al lado de la otra alrededor de este centro, y habitualmente están separadas en calles. Es importante notar que en estas aldeas se mezclan personas asháninkas de diferentes valles de la selva central, algunos con poco o sin ningún lazo de parentesco en las áreas en las que viven, a la cual Ilegaron escapando del conflicto interno a finales de los años 1980 y comienzos de los años 1990. Por ejemplo, Nueva Esperanza, la Comunidad Nativa del Bajo Urubamba, en la que hice parte de mi trabajo de campo, estaba compuesta por familias de los ríos Bajo Urubamba, Ucayali, y Tambo, pero también incluía familias de lugares más lejanos como el Gran Pajonal, Satipo, Río Negro, y los ríos Ene y Perené.

Mis colaboradores asháninkas subrayan el peligro potencial de tener un gran número de personas viviendo en estrecha proximidad, y notan, como se puede ver en las citas anteriores, que sus ancestros comunes no podían vivir juntos en aldeas porque se hubieran estado peleando constantemente. Si bien los asháninkas, al igual que muchas otras sociedades indígenas amazónicas (ver Santos-Granero, 2000), tienen mecanismos tradicionales para prevenir conflictos, como se ejemplifica en su conocida preferencia por separarse para no dejar que un conflicto se vuelva serio, no tienen mecanismos tradicionales para resolver conflictos. Sin embargo, esta separación no es viable hoy ya que los aldeanos no 
solo quieren vivir cerca de escuelas y postas médicas, sino que muchas de las casas en las aldeas en las que he vivido y visitado están construidas con materiales más caros y duraderos que las tradicionales (por ejemplo, ahora se utilizan maderas duras y techos de calamina), que son más difíciles de mover, y demasiado caras para abandonarlas. Cualquier mudanza se vuelve más complicada ya que las familias asháninkas están acumulando mucho más objetos materiales que en el pasado, y también están participando cada vez más en el cultivo de especies comerciales a largo plazo como el café y el cacao.

A pesar de esto, mis interlocutores asháninkas destacan que la Comunidad es clave para su búsqueda contemporánea del kametsa asaiki. Al preguntarle a Chato, uno de mis vecinos en Nueva Esperanza, qué significa ser un comunero (oficialmente, un miembro legal de la Comunidad Nativa), el me respondió:

Ser un comunero es poder vivir tranquilo. Vivir en una Comunidad es lindo (...) los antiguos vivían lejos y se visitaban a veces nomás para tomar masato. Vivían tristes. Ahora visitamos a nuestras familias todos los días para tomar masato, o a nuestros vecinos (...) Tomamos tranquilos, conversamos, hacemos chistes. Jugamos futbol, hacemos grupos grandes para hacer pesca, para limpiar nuestro lindero. iEs lindo, vivimos tranquilos!

De igual modo, Delfín, otro comunero de Nueva Esperanza, me dijo que ser un comunero es:

Vivir tranquilo, libre para trabajar mi chacra, libre para moverme en nuestro territorio, para hacer pesca con mi familia, para cazar (...) En la Comunidad se puede trabajar tranquilo, plantar tu cacao, tu café (...) no es como con un patrón que grita todo el día, diciendo «anda has esto, anda trae agua» (...) Nadie te dice qué hacer, puedes descansar y tomar masato después de trabajar en tu chacra, y después ir a tu casa a comer con tu familia. (...) cuando la gente vivía separada vivían mal, hacían guerra por cualquier cosa [...] era muy triste.

La proximidad física de la vida en las aldeas de hoy se ha convertido en la organización espacial ideal.

A esto debemos sumarle el gran deseo de mis colaboradores de vivir cerca de una escuela para que sus hijas e hijos asistan a ella. La escuela, como se ha notado antes en la zona (ver, por ejemplo, Gow, 1991), se convierte en una fuente de sabiduría «civilizada» que ellos dicen que les permite evitar el abuso por parte de los patrones madereros y caucheros que sus antepasados sufrieron:

Nuestros abuelos no vivían en [aldeas] por que no (...) sabían [vivir en aldeas]. Vivían lejos para no estar peleándose (...) pero ahora vivimos así juntos porque estamos organizados, vivimos todos tranquilos, podemos trabajar tranquilos y tomar masato. Antes no habían escuelas, la gente no sabía leer o escribir, entonces no entendían (...) Ahora todo está cambiando, tenemos nuestro título, sabemos cuánto se paga [mensualmente por trabajar para patrones madereros] y ya no nos hacen idiotas en Atalaya. Por eso es que es importante vivir en una Comunidad. 


\section{Alejo, un comunero asháninka que vivió en el fundo de un patrón mestizo en el río Ucayali cuando era niño, antes de mudarse a una Comunidad en los años 1980, me dijo que:}

Ahora los niños están aprendiendo en la escuela, por eso es bueno vivir en Comunidad. Los niños ya saben ya, no van a ser como los viejos que nunca aprendieron. Ahora quien menos puede ganarse algo [de dinero] para comprar sal, jabón (...) no es como antes con los patrones que nos abusaban por que no sabíamos leer, no sabíamos de nuestros derechos.

La Ley de Comunidades Nativas no solo ha llevado a un tipo de organización de la vida comunal que no existía entre las personas asháninkas, sino también una estructura política que va en contra del tipo de estructuras de autoridad que conocemos para el área etnográfica, sobre todo en lo que se refiere a los modelos de autoridad.

\section{ESTRUCTURAS DE LIDERAZGO ASHÁNINKA EN LOS TIEMPOS DE LA COMUNIDAD NATIVA}

El título de cada Comunidad Nativa está constituido colectivamente por sus comuneros, quienes están legalmente obligados a elegir una junta directiva cada dos años. Estas juntas deben ser lideradas por un jefe o una jefa, y deben ser inscritas en los registros públicos. Esta inscripción confirma que el Estado peruano reconoce al nuevo jefe como representante legal de la Comunidad.

France-Marie Renard-Casevitz (1993; ver también Brown, 1993) ha notado que en los primeros años de la Comunidad Nativa, se elegían a hombres jóvenes que podían leer y escribir en español, pero que no tenían verdadero ascendiente en la Comunidad ya que los otros comuneros no les dejaban ejercer ningún tipo de poder. En estos casos el verdadero poder de liderazgo quedaba aún con los lideres tradicionales. Es cierto que el poder de estas autoridades viene desde afuera (desde el Estado por medio de la Ley de Comunidades Nativas), y que a estos jefes y jefas no se les permite entrometerse en las cuestiones de las redes de parentesco que se forman en la vida cotidiana. Sin embargo, este tipo de análisis no toma en cuenta la importancia del poder legal que conlleva la firma del jefe.

Las lecturas críticas más recientes de la Comunidad (ver, por ejemplo, Greene, 2009) resaltan las ventajas que los líderes comunales tienen sobre otros comuneros por el poder legal que detentan sobre los recursos naturales dentro del territorio de la Comunidad. Las historias de jefes o jefas que terminan endeudados por miles de nuevos soles tras pedir préstamos o adelantos a empresas madereras locales son bien conocidas a través de toda la selva peruana. Greene (2009) describe cómo estas ventajas llevan a conflictos que se basan en la imposición de actitudes jerárquicas de liderazgo por parte de las autoridades que interfieren en la vida diaria de los comuneros. El potencial de abuso de la posición se manifiesta, en algunos casos, en las diferencias entre las casas de los jefes y las de otros comuneros, y el grado desigual de acumulación material entre ellos (ver, por ejemplo, Sarmiento Barletti, 2015). 
Como he notado antes para el caso asháninka (Sarmiento Barletti, 2016a), a estos jefes se les permite abusar de su puesto, y a veces acarrear deudas de miles de soles, siempre y cuando consigan beneficios para sus Comunidades y no se entrometan en la vida cotidiana de los otros comuneros. Estos jefes solo tienen algún tipo de autoridad en lo que corresponde a sus interacciones con el mundo exterior, o en temas internos que tengan que ver con la Comunidad. La Comunidad puede permitir a los líderes asháninkas actuar con más poder que el que se ve en los trabajos etnográficos sobre el liderazgo entre los asháninkas11, pero esto les brinda a los otros comuneros la protección, servicios, bienes materiales, y soporte organizacional que necesitan para su bienestar en un contexto tan complicado. Los trámites y convenios que logran las autoridades de la Comunidad les da a los comuneros un espacio legalmente protegido en el cual vivir con otras personas asháninkas, además de una escuela para sus hijos, una posta médica, y una serie de bienes materiales muy deseables (como, por ejemplo, botes, motores fuera de borda, y techos de calamina). Estos se pueden adquirir por medio de convenios con entes externos a la comunidad, como el estado, ONG nacionales y extranjeras, patrones madereros, y empresas de hidrocarburos. Mis interlocutores asháninkas manifiestan que la autonomía y prosperidad que se derivan de vivir en una Comunidad, se mantienen en balance con los poderes que reciben estos líderes. Como veremos en lo que sigue, la autoridad permitida a estos jefes en sus interacciones con agentes no asháninkas también se debe a cómo mis colaboradores desconectan la posición del jefe (y otras autoridades) de los lazos de parentesco diarios y de las responsabilidades que trae ser parte de estos nexos ${ }^{12}$.

\section{EL COMUNERO Y EL ASHÁNINKA SANORI}

Propongo que para entender esta desconexión, debemos centrar nuestro análisis en cómo la Comunidad Nativa permite la existencia paralela y estratégica de dos identidades diferentes: el asháninka sanori («persona legítima») y el comunero. Para ello me baso en la distinción similar que hace Dan Rosengren (2003) para los matsigenkas, vecinos de los asháninkas, río arriba en el Bajo Urubamba. Siguiendo a Gilles Deleuze \& Felix Guattari (1988), Rosengren propone que las sociedades indígenas amazónicas pueden seguir dos modelos distintos para la construcción de su identidad. El primero es el modelo «raíz base», un sistema arbitrario que hace hincapié en el «origen como único, y la cultura como identificada con la etnicidad»(Rosengren, 2003: 223, mi traducción). Este modelo puede

11 Los textos sobre el tema analizan el fervor con el que los asháninkas intentan prevenir que sus líderes se impongan sobre los otros miembros del grupo (Hvalkof \& Veber, 2005; Killick, 2005; 2007). De hecho, los textos describen que, como en otras sociedades vecinas (ver Rosengren, 1987 para un análisis similar entre los matsigenkas), los grupos asháninkas raramente le dan mucha autoridad a un individuo, y normalmente esto solo se hace en casos de guerra.

12 A diferencia de lecturas clásicas sobre el liderazgo y autoridad en la región (ver, por ejemplo, Brown, 1993 y Overing, 2012) el poder de autoridad ya no recae sobre el individuo, sino sobre la institución. Este es un tema para desarrollar en el futuro. 
usar autodenominaciones indígenas, pero en formas que van de acuerdo con «nociones geopolíticas occidentales que asumen la existencia de un lazo orgánico entre personas, lugar, e idioma» (Rosengren, 2003: 223, mi traducción). El modelo raíz base también se puede aplicar a la conceptualización de pertenecer a una Comunidad Nativa como una entidad política, ya que aunque no todos los comuneros de una Comunidad están emparentados, sí están relacionados por sus derechos legales sobre un territorio común sobre el cual viven. El título sobre este territorio les fue otorgado por el Estado peruano, basado en un criterio étnico. La participación de los comuneros en asambleas comunales y el uso de las tierras de la Comunidad Nativa, confirman que todos son comuneros.

Rosengren también propone que las autodenominaciones indígenas también pueden ser entendidas por medio de un modelo «rizomático» que enfatiza

la comunidad local (...) donde las relaciones concretas son lo que vale (Rosengren, 2003: 224, mi traducción).

Los miembros de estas agrupaciones rizomáticas se relacionan a través de lazos de parentesco que se establecen por medio de relaciones de confianza y cuidado que se esfuerzan en construir en el día a día como parte de su búsqueda del kametsa asaiki. Entonces, mientras que el modelo raíz base se asienta en la noción de pertenecer a la Comunidad como un espacio político, el modelo rizomático se basa en la noción de pertenecer a una de las parentelas que viven dentro de ese espacio físico. Podemos asociar a la Comunidad con la identidad del comunero, asumida por los que viven dentro del territorio titulado, y las unidades de parentesco con la identidad del asháninka sanori, asumida por las personas asháninkas en sus interacciones diarias.

Ahora, estas categorías de pertenencia a un grupo no son mutuamente exclusivas. Son dos modelos que un individuo puede adoptar y utilizar en diferentes contextos. Propongo que la razón por la cual la Comunidad se vuelve tan clave para el kametsa asaiki es porque, al hacer el cambio estratégico entre las identidades de comunero y asháninka sanori, mis colaboradores pueden enfrentarse a situaciones que de otra forma serían muy complicadas para lidiar de manera interna, y hubieran provocado fisiones dentro de sus comunidades en el pasado. Aunque estos dos modelos identitarios sean muy porosos, propongo que también sirven como tipos ideales para el análisis que planteo en este artículo.

La separación entre el modelo del comunero y el asháninka sanori se hace más obvia en las asambleas comunales, que normalmente se llevan a cabo en la casa comunal de la Comunidad. Esta edificación representa una frontera simbólica entre los asháninkas que viven dentro de una Comunidad y el exterior. Es en la casa comunal donde se recibe a los visitantes a la Comunidad, donde se iza la bandera peruana, y donde se llevan a cabo los otros eventos formales relacionados con la Comunidad. Las asambleas comunales reflejan el entendimiento de los tratos oficiales de la Comunidad. Los tíos, cuñados, hermanas, o sobrinas, dejan de relacionarse como tal para convertirse en jefes, presidente del comité de autodefensa, o secretario. Este cambio de modelo identitario marca un límite simbólico entre la vida cotidiana dentro de la aldea y la vida formal de la Comunidad. 
Las asambleas comunales son representaciones ritualizadas que invierten la vida cotidiana: se escriben actas sobre los temas de la reunión y al fin de esta se llega a un acuerdo que todos los presentes firman, y las autoridades sellan. En las asambleas comunales los participantes visten sus mejores ropas, hablan formalmente y utilizan sus títulos (por ejemplo, jefe, vice-jefe, vocal, etc.) en vez de los términos de parentesco que se usan en la vida cotidiana, y cada uno espera en turno a que la autoridad que dirige la asamblea lo llame para poder hablar. El relacionarse como comuneros en vez de como asháninka sanori permite que estas asambleas se conviertan en espacios para la externalización de rondas de chismes que tratan diferentes tipos de actos anti-convivenciales en la aldea (como, por ejemplo, robos o violencia doméstica). Esto lleva a que las asambleas se conviertan en un contexto en el que no sea muy raro que un comunero avergüence públicamente a otro, o que se vean arrebatos de ira en medio de fuertes discusiones. Este tipo de actitudes son muy raras en la vida cotidiana. Las mujeres, que normalmente no participan en los debates, se sientan a un lado, como una especie de coro, y comentan de manera muy crítica los problemas de la Comunidad, expresando lo que piensan sobre el accionar incorrecto o inconveniente de sus autoridades comunales.

También he participado en asambleas comunales en las que alguna autoridad comunal, a pedido de un comunero, aborda públicamente temas de robos, peleas, o adulterio. Sin embargo, esto se hace por medio de «consejos» que, como me explicó uno de mis colaboradores, son tan serios y vienen con tal autoridad, que hacen que la gente «tenga vergüenza» o se «acuerde como sentir vergüenza» por sus actos. El lidiar con estos problemas dentro de un espacio micropolítico como son las asambleas comunales se hace necesario porque los asháninkas son adversos al conflicto en la vida cotidiana. Una vez terminada la asamblea comunal, los participantes se relacionan de nuevo como asháninka sanori, y se vuelven a separar en las parentelas de las que son parte en la vida cotidiana. Los cambios estratégicos entre los modelos identitarios de asháninka sanori y comunero son claves en el contexto de las asambleas. En efecto, un asháninka sanori no puede decirle a otro ser autónomo qué hacer, mientras que los comuneros requieren que sus autoridades mantengan y salvaguarden el orden político, y consigan los bienes materiales y servicios que son necesarios para seguir con la búsqueda del kametsa asaiki.

\section{CONCLUSIÓN: CÓMO UTILIZAR A LA COMUNIDAD PARA DEFENDER A LA COMUNIDAD}

Hvalkof \& Veber (2005) describen el sistema social asháninka como muy flexible, en el cual la fragmentación y la ruptura son comunes. Sin embargo, también subrayan que se pueden dar uniones y acciones colectivas momentáneas en diferentes contextos. No obstante, la forma en la que mis interlocutores asháninkas abordan y experimentan la Comunidad Nativa parece permitir que estas uniones vayan a durar por mucho más tiempo en algunas áreas, en especial 
en reacción a los peligros presentados por los proyectos extractivos que afectan la búsqueda del kametsa asaiki. Al separar la comunidad de asháninka sanori de los tratos oficiales de la Comunidad Nativa, los asháninkas con los que trabajo han logrado aprovecharse de las estructuras que trae esta figura legal para vivir juntos y defender el modo de vida que ellos desean y sus territorios de las figuras poderosas interesadas en los recursos naturales dentro de estos. La Comunidad Nativa también les permite aprovecharse de los beneficios de tener un nuevo tipo de jefe con poder legal para interactuar con otras figuras poderosas y lograr beneficios materiales y legales para reforzar la búsqueda del kametsa asaiki. Este artículo ha sido tan solo un pequeño ejemplo de cómo mis colaboradores asháninkas han tomado una figura legal que parecía un proyecto etnocida encubierto, y lo han remodelado para que quepa dentro de su visión del mundo, su enfoque sobre lo social, y su proyecto de bienestar.

\section{Referencias citadas}

BARCLAY, F. \& SANTOS-GRANERO, F., 1980 - La Conformación de las Comunidades Amuesha: La Legalización de Un Despojo Institucionalizado. Amazonía Peruana, 3 (5): 43-74.

BELAúNDE TERRY, F., 1959 - La conquista del Perú por los peruanos, 175 pp.; Lima: Ediciones Tahuantinsuyo.

BELAUNDE, L. E., 2000 - The Convivial Self and the Fear of Anger amongst the AiroPai of Amazonian Peru. In: The Anthropology of Love and Anger: The Aesthetics of Conviviality in Native Amazonia (J. Overing \& A. Passes, eds.): 209-221; Londres: Routledge.

BROWN, M., 1993 - Facing the State, Facing the World: Amazonia's Native Leaders and the New Politics of Identity. L'Homme, 33: 307-326.

CARUSO, E., 2012 - Being at the Centre: Self and Empire among Ene Ashaninka People in Peruvian Amazonia; University of Kent, Reino Unido. Tesis doctoral.

CHIRIF, A., 1980 - Colonización e Invasión: el Despojo Institucionalizado. Amazonía Indígena, 1 (1): 15-24.

CHIRIF, A., 1982 - Crónica de un atropello mal programado. Amazonía Indígena, 4: 3-11.

CHIRIF, A. \& GARCÍA HIERRO, P., 2007 - Marcando territorio: progresos y limitaciones de la titulación de territorios indígenas en la Amazonía, 340 pp.; Copenhagen: IWGIA.

CLASTRES, P., 1987 [1974] - Society Against the State. Essays in political anthropology, 218 pp.; New York: Zone Books.

COMISIÓN DE LA VERDAD Y DE LA RECONCILIACIÓN-CVR, 2002 - Informe Final, Lima: Comisión de la Vedad y de la Reconciliación.

DELEUZE, G. \& FÉLIX GUATTARI, 1988 - A Thousand Plateaus: Capitalism and Schizophrenia, xix + 610 pp.; Londres: Athlone.

DENEVAN, W., 1974 - Campa Subsistence in the Gran Pajonal, Eastern Peru. Geographical Review, 61: 496-518. 
ESPINOSA, O., 2010 - Cambios y continuidades en la percepción y demandas indígenas sobre el territorio en la Amazonía Peruana. Anthropologica, 28 (28): 239-262.

GOW, P., 1991 - Of Mixed Blood: Kinship and History in Peruvian Amazonia, x + 331 pp.; Oxford: Claredon Press.

GOW, P., 2000 - Helpless - The Affective Preconditions of Piro Social Life. In: The Anthropology of Love and Anger: The Aesthetics of Conviviality in Native Amazonia (J. Overing \& A. Passes, eds.): 46-63; Londres: Routledge.

GRAY, A., 1997 - Indigenous Rights and Development: Self-Determination in an Amazonian Community, xxi + 354 pp.; Eastbourne: Berghahn Books.

GRAY, A., 1998 - Demarcating Development: Titling Indigenous Territories in Peru. In: Liberation through Land Rights in the Peruvian Amazon (P. García Hierro, S. Hvalkof \& A. Gray, eds.): 165-215; Copenhagen: IWGIA.

GREENE, S., 2009 - Customizing Indigeneity: Paths to a Visionary Politics in Peru, xvi + 244 pp.; Palo Alto: Stanford University Press.

HARVEY, D., 2003 - The New Imperialism, ix + 253 pp.; Oxford: Oxford University Press.

HVALKOF, S., 1998 - From Slavery to Democracy: The Indigenous Process of Upper Ucayali and Gran Pajonal. In: Liberation through Land Rights in the Peruvian Amazon (P. García Hierro, S. Hvalkof \& A. Gray (eds.): 83-162; Copenhagen: IWGIA.

HVALKOF, S. \& VEBER, H., 2005 - Los Asheninka del Gran Pajonal. In: Guía etnográfica de la Alta Amazonía. Volumen V: Campa Ribereños/Ashéninka (F. Santos-Granero \& F. Barclay, eds.): 75-279; Lima: IFEA.

KILLICK, E., 2005 - Living Apart: Separation and Sociality amongst the Asheninka of Peruvian Amazonia; Londres: London School of Economics. Tesis doctoral.

KILLICK, E., 2007 - Autonomy and Leadership: Political Formations among the Ashéninka of Peruvian Amazonia. Ethnos, 72 (4): 461-482.

KILLICK, E., 2008 - Creating community: land titling, education and settlement formation amongst the Ashéninka of Peruvian Amazonia. Journal of Latin American and Caribbean Anthropology, 13 (1): 22-47.

OVERING, J., 2003 - In Praise of the Everyday: Trust and the Art of Social Living in an Amazonian Community. Ethnos, 68 (3): 293-316.

OVERING, J., 2012 - The Spectre of the Tyrant: Power, Violence and the Poetics of an Amazonian Egalitarianism. In: Contesting the State: The Dynamics of Resistance and Control (A. Hobart \& B. Kapferer, eds.): 55-85; Wantage: Sean Kingston.

OVERING, J. \& PASSES, A., 2000 - Introduction: Conviviality and the Opening Up of Amazonian Anthropology. In: The Anthropology of Love and Anger: The Aesthetics of Conviviality in Native Amazonia (J. Overing \& A. Passes, eds.): 1-30; Londres: Routledge.

PIMENTA, J., 2006 - Reciprocidade, mercado e desigualdade social entre os Ashaninka do Rio Amonia; Brasilia: Univerdidade de Brasilia. Serie Antropologica.

RENARD-CASEVITZ, F.-M., 1993 - Guerriers du sel, sauniers de la paix. L'Homme, 33: 126-128.

ROSENGREN, D., 1987 - In the Eyes of the Beholder: Leadership and the Social Construction of Power and Dominance among the Matsigenka of the Peruvian Amazon, viii + 231 pp.; Göteborgs: Göteborgs Etnografiska Museum.

ROSENGREN, D., 2003 - The Collective Self and the Ethno-Political Movement: 'Rhizomes' and 'Taproots' in the Amazon. Identities: Global Studies in Culture and Power, 10 (2): 221-240. 
SANTOS-GRANERO, F. 2000 - The Sisyphus Syndrome, or the Struggle for Conviviality in Native Amazonia. In: The Anthropology of Love and Anger: The Aethetics of Conviviality in Nativa Amazonia (J. Overing \& A. Passes, eds.): 268-287; Londres: Routledge.

SANTOS-GRANERO, F. \& BARCLAY, F., 1998 - Selva Central: History, Economy, and Land Use in Peruvian Amazonia. Washington, DC: Smithsonian Institute.

SARMIENTO BARLETTI, J. P., 2011 - Kametsa Asaiki: The Pursuit of the "Good Life" in an Ashaninka Village (Peruvian Amazonia); St. Andrew: University of St Andrews. Tesis doctoral.

SARMIENTO BARLETTI, J. P., 2015 - 'It makes me sad when they say we are poor, we are rich!': of wealth and public wealth in indigenous Amazonia. In: Images of Public Wealth in Tropical America (F. Santos-Granero, ed.): 139-160; Tucson: University of Arizona Press.

SARMIENTO BARLETTI, J. P., 2016a en prensa - The rise of the egalityrant in post-war Peruvian Amazonia. In: Creating Dialogues: Indigenous Perceptions and Forms of Leadership in Amazonia (P. Virtanen \& H Veber, eds.); Boulder: University of Colorado Press.

SARMIENTO BARLETTI, J. P., 2016b, en prensa - The Angry Earth: Wellbeing, Place, and Extractive Development in the Amazon. Anthropology in Action, 23 (3).

SURRALLÉS, A. \& GARCÍA HIERRO, P. (eds.), 2005 - The Land Within. Indigenous Territory and Perception of the Environment, 277 pp.; Copenhague: IWGIA.

TAYLOR, A. C., 1996 - The Soul's Body and Its States: An Amazonian Perspective on the Nature of Being Human. Journal of the Royal Anthropological Institute, 2 (2): 201-215.

VEBER, H., 1998 - The Salt of the Montana: Interpreting Indigenous Activism in the Rain Forest. Cultural Anthropology, 13 (3): 382-413.

WEISS, G., 1975 - Campa Cosmology: The World of a Forest Tribe in South America, 380 pp.; New York: American Museum of Natural History. 